\title{
Índice cárdio-femoral para avaliação da anemia de fetos de gestantes isoimunizadas
}

\author{
Cardiofemoral index for the evaluation of fetal anemia in isoimmunized pregnancies \\ Antônio Carlos Vieira Cabral ${ }^{1}$, Thales Bittencourt de Barcelos², Isabela Gomes Melo Apocalipse ${ }^{2}$, \\ Henrique Vitor Leite ${ }^{3}$, Zilma Silveira Nogueira Reis ${ }^{2}$
}

\section{RESUM0}

Objetivo: estabelecer novo marcador não invasivo na detecção da anemia fetal, em gestantes isoimunizadas por antígenos eritrocitários. Métodos: em estudo transversal o índice ecográfico obtido pela razão entre a medida ecográfica do diâmetro biventricular externo (DBVE) e do comprimento do fêmur (ambos em centímetros) foi correlacionado à concentração da hemoglobina fetal. Foram incluídos no estudo 59 fetos de gestantes isoimunizadas selecionados para propedêutica invasiva que foram submetidos a 130 cordocenteses para diagnóstico e tratamento da anemia. A medida do índice cárdio-femoral foi obtida imediatamente antes da cordocentese e o valor da hemoglobina foi determinado em amostra de sangue fetal obtida durante o procedimento. A correlação entre o índice cárdio-femoral e o valor da hemoglobina fetal foi avaliada por regressão linear e o ponto de corte de melhor acuidade no diagnóstico da anemia (hemoglobina menor que $10 \mathrm{~g} / \mathrm{dL}$ ), verificado pela curva ROC. Resultados: a medida ecográfica do DBVE variou de 1,6 a 4,7 cm (média 2,5 $\pm 1,3 \mathrm{~cm}$ ) e a do comprimento do fêmur

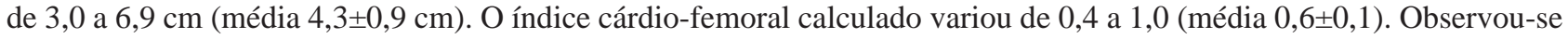
correlação inversa entre o índice ecográfico e a hemoglobina fetal $\left(\mathrm{R}^{2}=0,37 \mathrm{e} \mathrm{p}<0,0001\right)$. O valor 0,60 para o índice cárdiofemoral foi o ponto de corte com melhor acuidade para a predição de um nível de hemoglobina fetal menor que 10,0 g/dL: sensibilidade $80,9 \%$, especificidade $83,1 \%$, valor preditivo positivo $73,1 \%$ e valor preditivo negativo $88,5 \%$. Conclusão: 0 índice cárdio-femoral apresentou boa acuidade na predição do nível de hemoglobina fetal abaixo de $10 \mathrm{~g} / \mathrm{dL}$ em gestantes isoimunizadas por antígenos eritrocitários, podendo se tornar marcador não invasivo da anemia fetal.

PALAVRAS-CHAVE: Isoimunização RH; Anemia neonatal/diagnóstico; Ultrasonografia/métodos; Análise transversal

\section{ABSTRACT}

Purpose: to test a new, noninvasive method for the diagnosis of fetal anemia in red blood cell isoimmunized pregnancies. Methods: the index obtained by the ratio between the ultrasonographic measurement of the biventricular outer dimension (BVOD) and femur length (both in centimeters) was correlated with fetal hemoglobin values in a cross-sectional study. Fiftynine fetuses of isoimmunized pregnancies selected for invasive treatment and submitted to 130 cordocenteses for the diagnosis and treatment of anemia were included in the study. The cardiofemoral index was obtained immediately before the cordocentesis and the fetal hemoglobin index was obtained from fetal blood samples. Linear regression was carried out to assess the correlation between the index and fetal hemoglobin; ROC curve was applied to determine the most accurate cutoff for the diagnosis of the fetal hemoglobin concentration below 10g/dL. Results: BVOD measurement varied from 1.6 to $4.7 \mathrm{~cm}$ (average $2.5 \pm 1.3 \mathrm{~cm}$ ), and length of the femur, from 3.0 to $6.9 \mathrm{~cm}$ (average $4.3 \pm 0.9 \mathrm{~cm}$ ). The cardiofemoral index varied from 0.4 to 1.0 (average $0.6 \pm 0.1$ ). A significant inverse correlation between the cardiofemoral index and fetal hemoglobin $\left(\mathrm{R}^{2}=0.37\right.$ and $\left.\mathrm{p}<0.0001\right)$ was observed. The cutoff of 0.60 was the best to predict a level of fetal hemoglobin below or equal to $10.0 \mathrm{~g} / \mathrm{dL}$ : $80.85 \%$ sensitivity, $83.13 \%$ specificity, $73.8 \%$ positive predictive value, and $88.46 \%$ negative predictive value, in the diagnosis of fetuses anemia. Conclusion: the cardiofemoral index allows for good accuracy in the prediction of fetal hemoglobin concentration below $10 \mathrm{~g} / \mathrm{dL}$ in red blood cell isoimmunized pregnancies. It may thus be applied as a noninvasive method to the diagnosis of this pathology.

KEYWORDS: RH Isoimmunization; Anemia, neonatal/diagnosis; Ultrasonography/methods; Period analysis

1 Professor Titular do Departamento de Ginecologia e Obstetrícia da Universidade Federal de Minas Gerais - UFMG - Belo Horizonte (MG), Brasil.

2 Médico do Hospital das Clínicas da Universidade Federal de Minas Gerais - UFMG - Belo Horizonte (MG), Brasil.

3 Professor Adjunto do Departamento de Ginecologia e Obstetrícia da Universidade Federal de Minas Gerais - UFMG - Belo Horizonte (MG), Brasil.

Centro de Medicina Fetal do Hospital das Clínicas da Universidade Federal de Minas Gerais

Correspondência: Antônio Carlos Vieira Cabral

Centro de Medicina Fetal do Hospital das Clínicas da Universidade Federal de Minas Gerais

Av. Alfredo Balena, 190, 4º andar - 30130-100 - Belo Horizonte-MG - Fone/FAX: (031) 3248-9421 / 9991-8256 - e-mail: accabral@hc.ufmg.br

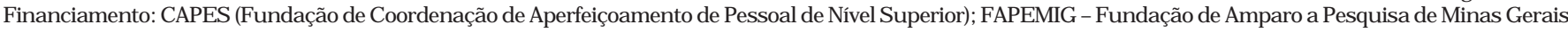




\section{Introdução}

A anemia fetal é ainda uma importante intercorrência do período antenatal. As causas são diversas e, nos países em desenvolvimento, destaca-se a isoimunização materna aos antígenos eritrocitários, principalmente aqueles do complexo $\mathrm{Rh}^{1,2}$.

O seu diagnóstico é tradicionalmente realizado por meio de procedimentos invasivos: a amniocentese, para estudo da concentração de bilirrubina no líquido amniótico, e a cordocentese, para avaliação hematimétrica fetal $^{3}$. Embora considerados relativamente seguros, os procedimentos invasivos podem se acompanhar de complicações graves como a punção arterial, fator de risco para bradicardia e sofrimento fetal ${ }^{4}$, o que justifica a busca de parâmetros não invasivos que possam predizer com segurança a intensidade da anemia fetal.

A propedêutica ultra-sonográfica antenatal vem mostrando avanços no diagnóstico indireto da anemia fetal, por meio da dopplerfluxometria da artéria cerebral média ${ }^{5,6}$ e da aorta abdominal ${ }^{7}$. Também têm sido relevantes como parâmetros indiretos do estado anêmico as medidas ecográficas como medidas do tamanho da placenta, do baço e do fígado ${ }^{8} \mathrm{e}$ o diâmetro da veia umbilical ${ }^{9}$.

Além disto, avaliações oriundas da ecocardiografia fetal têm se mostrado promissoras como marcadores não invasivos na predição da anemia fetal. A medida do diâmetro biventricular externo (DBVE) é método propedêutico de grande acurácia na detecção da cardiomegalia fetal ${ }^{10}$. Foi observada correlação inversa entre a concentração da hemoglobina no sangue fetal e a medida do DBVE, independente da idade gestacional ${ }^{11}$, o que abriu perspectivas para seu emprego na predição dos índices hematimétricos fetais.

O valor da hemoglobina fetal é considerado crítico para indicação de transfusão intra-uterina quando se situa abaixo de 10-11 g/dL com hematócrito abaixo de $0,30^{2}$, considerados no contexto da história obstétrica anterior, título de anticorpos e a valores da espectrofotometria de líquido amniótico. A determinação de um parâmetro indireto seguro de avaliação da anemia fetal correspondente a este limiar hematimétrico seria de grande utilidade na expectativa de redução da morbidade e mortalidade associadas aos procedimentos invasivos. Embora esta seja meta importante na propedêutica obstétrica atual, ainda não existe consenso de que seja possivel no momento, a substituição da amniocentese e da cordocentese por métodos não invasivos. O presente estudo pretende contribuir para esta busca, avaliando um novo marcador para o diagnóstico da anemia fetal em gestantes isoimunizadas por antígenos eritrocitários.

\section{Métodos}

Em estudo transversal observacional realizado entre janeiro de 2002 e junho de 2003, foram incluídos 59 fetos de gestantes portadoras de isoimunização por antígenos eritrocitários acompanhadas no Centro de Medicina Fetal do Hospital das Clínicas da Universidade Federal de Minas Gerais. As pacientes foram informadas sobre o protocolo de acompanhamento pré-natal e realização do estudo e concordaram voluntariamente com a participação, assinando o termo de consentimento. O estudo foi aprovado pelo Comitê de Ética do Hospital.

Foram considerados como critérios de inclusão a idade gestacional maior que 20 semanas e a existência de indicação de cordocentese para avaliação do estado de anemia fetal: valor do teste de Coombs indireto com titulação superior a 1:8, espectrofotometria do líquido amniótico na zona I da curva de Liley ${ }^{12}$ e/ou passado obstétrico desfavorável (perda de feto ou neonato anterior pela doença). A presença de doenças hipertensivas ou diabete materno (dois casos) e a existência de malformações fetais (um caso) foram consideradas para exclusão. A idade materna variou entre 19 e 34 anos (média 27,1 anos).

Para determinação da concentração da hemoglobina fetal foram realizadas 130 cordocenteses nos casos suspeitos de hemólise grave, sendo de 2,2 a média de procedimentos por feto. A hidropisia já estava presente antes do procedimento invasivo em 54 (41,5\%) casos e ausente em 76 (58,5\%). A idade gestacional no momento da cordocentese variou entre 21 e 34 semanas (média 28,6 semanas).

Todos os casos foram submetidos a ultrasonografia em modo-B e modo-M por médico do Centro de Medicina Fetal do hospital universitário até seis horas antes da cordocentese. O equipamento ultra-sonográfico e para dopplerfluxometria empregado foi Sonoace 8800 (Medson) com sonda de $3,5 \mathrm{MHz}$ e filtro acústico de $100 \mathrm{~Hz}$, e monitor fetal anteparto HP-50. A medida do DBVE foi obtida segundo técnica descrita por DeVore et al. $(1985)^{13}$. Obteve-se a imagem ecográfica de quatro câmaras do coração fetal em tempo real e o cursor do modo $\mathrm{M}$ foi posicionado perpendicularmente ao septo interventricular no plano das 
valvas atrioventriculares. Para medida do DBVE, congelou-se a imagem na diástole cardíaca e mediu-se a distância entre os epicárdios dos ventrículos direito e esquerdo (Figura 1). A seguir, realizou-se a medida do comprimento do fêmur ao longo da diáfise, excluindo-se a epífise distal ${ }^{14}$. O indice cárdio-femoral foi calculado pela razão entre as medidas do DBVE e comprimento do fêmur.

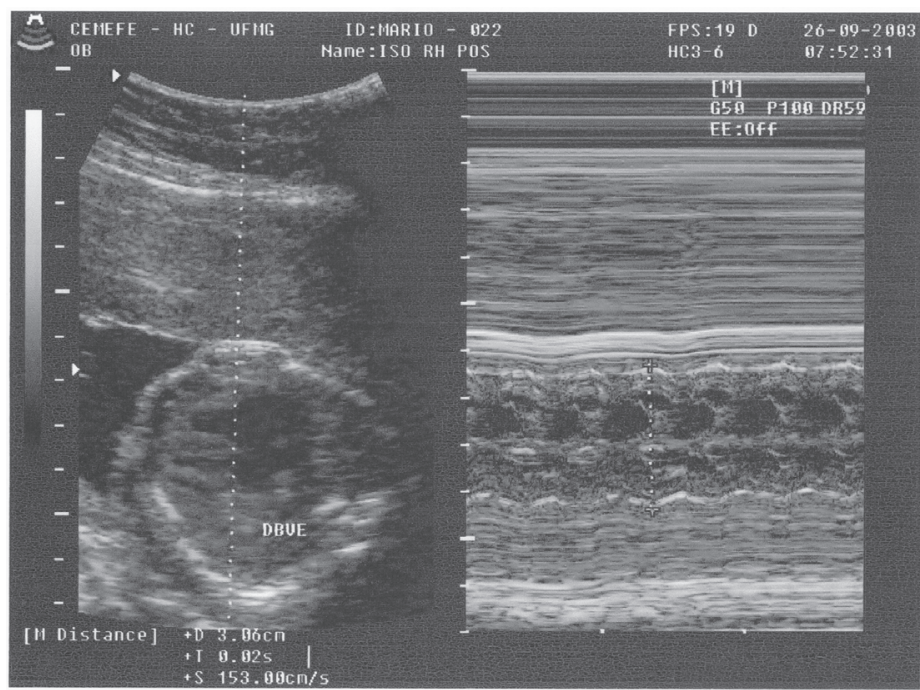

Figura 1 - Medida ultra-sonográfica do diâmetro biventricular externo (DBVE), pelo modo $\mathrm{M}$.

Uma amostra de sangue fetal obtida na cordocentese, cerca de $1 \mathrm{~mL}$, foi utilizada para análise hematimétrica. Uma gota de sangue foi empregada na dosagem da concentração de hemoglobina em equipamento de microdosagem Hemocue $^{\circledR}$ e o restante enviado para determinação do volume corpuscular médio por técnica convencional. Considerou-se o volume corpuscular médio maior que $108 \mathrm{fL}$ como indicativo de que a amostra de sangue colhida é de origem fetal, segundo protocolo do serviço ${ }^{2}$.

Em análise transversal, a associação entre o índice cárdio-femoral calculado e a concentração de hemoglobina fetal foi avaliada pela análise de regressão dos mínimos quadrados. Empregouse o programa Minitab Release, versão 11.12, considerando-se significativo um $\mathrm{p}<0,05$.

A seguir avaliou-se a acuidade diagnóstica do índice cárdio-femoral para a detecção da concentração da hemoglobina inferior a $10 \mathrm{~g} / \mathrm{dL}$, que é indicativa de transfusão intra-uterina no serviço $^{1,2}$. Esta acuidade foi pesquisada nos valores entre 0,50 a 0,66 por meio de medidas de validação de testes diagnósticos (sensibilidade, especificidade, valor preditivo positivo, valor preditivo negativo) e o ponto de corte ideal do índice cárdiofemoral para identificar o valor crítico de hemoglobina fetal inferior a $10 \mathrm{~g} / \mathrm{dL}$ foi determinado pela análise da curva ROC (receiver operator characteristic).

\section{Resultados}

A presença de concentração de hemoglobina fetal inferior a $10 \mathrm{~g} / \mathrm{dL}$, no momento da cordocentese, foi detectada em 47 casos $(36,2 \%)$. Os niveis de hemoglobina fetal variaram de 2,3 a $16,4 \mathrm{~g} / \mathrm{dL}$ (média 8,9 $\pm 4,6 \mathrm{~g} / \mathrm{dL}$ ). Acredita-se que não houve punção inadvertida de vaso materno, uma vez que todas as medidas de volume corpuscular médio das amostras de sangue indicavam valores superiores a $108 \mathrm{fL}$.

A medida ecográfica do DBVE variou de 1,6 a $4,7 \mathrm{~cm}$ (média $2,5 \pm 1,3 \mathrm{~cm}$ ) e a do comprimento do fêmur, de 3,0 a $6,9 \mathrm{~cm}$ (média $4,3 \pm 0,9 \mathrm{~cm}$ ). O índice cárdio-femoral calculado variou de 0,4 a 1,0 (média 0,6 $\pm 0,1$ ).

Observou-se correlação inversa significativa entre o índice cárdio-femoral e a hemoglobina fetal $\left(R^{2}=0,37\right.$ e $p<0,0001$ - Figura 2). Dessa forma constatou-se que à medida que a anemia se agrava, o que foi avaliado neste estudo pela diminuição da concentração de hemoglobina, o índice cárdio-femoral se eleva.

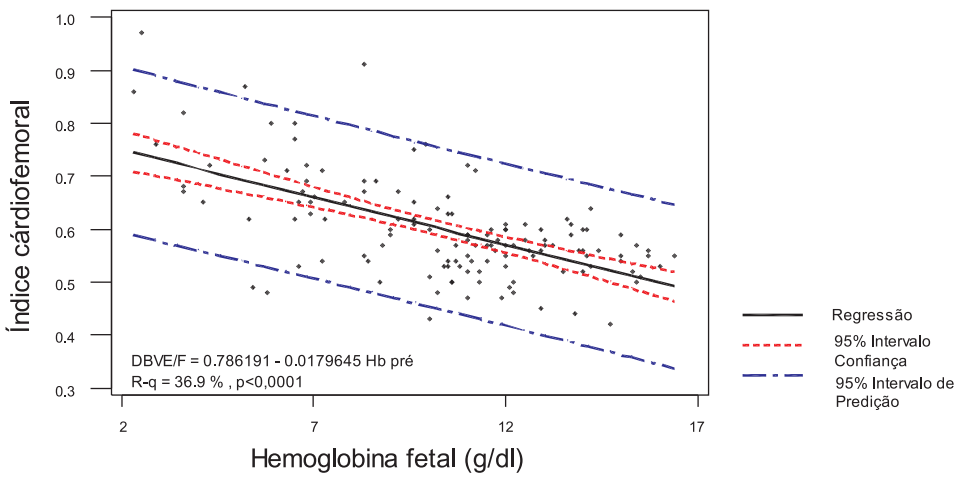

Figura 2 - Reta de regressão da correlação entre o índice cárdio-femoral e a hemoglobina fetal $(\mathrm{g} / \mathrm{dL})$. DBVE = diâmetro biventricular externo/comprimento do fêmur.

As medidas de validação do índice cárdiofemoral pelo cálculo da sensibilidade, especificidade, valor de predição positiva e valor de predição negativa, no diagnóstico da anemia fetal com nível de hemoglobina inferiores a $10 \mathrm{~g} / \mathrm{dL}$, são apresentadas na Tabela 1. O ponto de corte do índice cárdio-femoral com melhor acuidade para identificação do limiar de hemoglobina fetal proposto (10 $\mathrm{g} / \mathrm{dL}$ ) foi evidenciado na curva de sensibilidade $\mathrm{X}$ especificidade no valor de 0,6 (Figura 3). Neste ponto, 38 das 47 situações em que havia anemia 
clinicamente relevante ( $\mathrm{Hb}$ inferior a $10 \mathrm{~g} / \mathrm{dL})$ apresentavam o indice maior ou igual a 0,6 e 69 das 83 situações sem anemia relevante mostraram o índice menor que 0,6 , correspondendo a sensibilidade $80,9 \%$, especificidade $83,1 \%$, valor de predição positiva $73,1 \%$ e valor de predição negativa $88,5 \%$ (Tabela 1 ).

Tabela 1 - Acuidade diagnóstica do índice cárdio-femoral para a detecção de nível da hemoglobina fetal inferior a $10 \mathrm{~g} / \mathrm{dL}$, considerando-se pontos do índice entre 0,52 a 0,66 .

Índice Sensibilidade Especificidade Valor preditivo Valor preditivo

\begin{tabular}{ccccc} 
cárdio-femoral & $\mathbf{( \% )}$ & $\mathbf{( \% )}$ & positivo (\%) & negativo (\%) \\
\hline 0,52 & 93,6 & 22,9 & 40,7 & 86,4 \\
0,54 & 91,5 & 37,3 & 45,7 & 88,9 \\
0,56 & 85,1 & 50,6 & 49,4 & 85,7 \\
0,58 & 83,0 & 66,7 & 60,9 & 87,9 \\
0,60 & 80,9 & 83,1 & 73,1 & 88,5 \\
0,62 & 71,7 & 89,3 & 80,5 & 73,0 \\
0,64 & 63,8 & 95,2 & 88,2 & 82,3 \\
0,66 & 53,2 & 96,4 & 89,3 & 78,4 \\
\hline
\end{tabular}

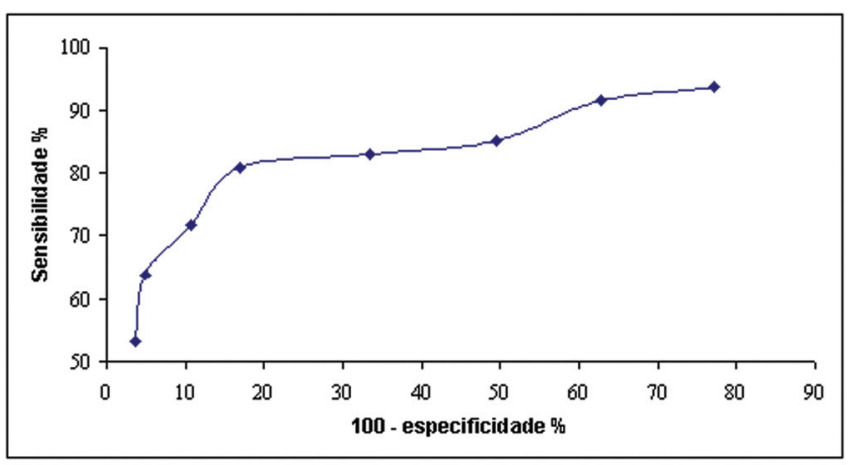

Figura 3 - Curva sensibilidade versus especificidade para detecção de fetos com anemia (nível de hemoglobina inferior a $10 \mathrm{~g} / \mathrm{dL}$ ), com 0 índice cárdio-femoral.

\section{Discussão}

A isoimunização materna pelo fator Rh é ainda uma realidade em nosso país e os centros universitários os serviços de referência no diagnóstico e tratamento desta doença. Diferentes métodos não invasivos para predição da anemia fetal estão hoje disponíveis, com graus variáveis de precisão, de acordo com o tipo do teste empregado e definição da anemia fetal. A grande vantagem desses métodos para a monitorização de fetos comprometidos pela doença hemolítica perinatal é a redução da exposição materno-fetal aos riscos da amniocentese e da cordocentese.

Métodos de avaliação fetal como a cardiotocografia têm importante papel na condução das gestações isoimunizadas ${ }^{15}$, todavia seu valor é reduzido antes da $28^{\mathrm{a}}$ semana de gestação devido à imaturidade da inervação do coração fetal. Considerando as modificações adaptativas do feto à anemia, a ecografia e a dopplerfluxometria, por meio da detecção das alterações biométricas e hemodinâmicas, possuem grande potencial para a identificação desse processo patológico ${ }^{3}$.

A dopplerfluxometria dos vasos sanguíneos fetais é apontada como método promissor nos protocolos de acompanhamento dos fetos anêmicos, mas ela requer maior habilidade técnica, equipamentos mais caros e está mais sujeita a resultados imprecisos do que os exames biométricos, devido a movimentos respiratórios e somáticos do feto. Boa acuidade para predição da anemia fetal pela dopplerfluxometria vem sendo obtida, mas especialmente em casos de grandes déficits de hemoglobina ${ }^{5,16}$. Sensibilidade de $75 \%$ e especificidade de $60 \%$ foram obtidas no estudo da artéria cerebral média no diagnóstico da anemia moderada $^{17}$, permanecendo a lacuna de um método seguro para o diagnóstico da anemia leve a moderada, pois sabe-se que o ideal é a realização do tratamento intra-uterino com transfusão antes que o feto apresente déficits muito grandes de hemoglobina e se torne hidrópico. No presente estudo, procuramos identificar situações em que os fetos apresentavam anemia moderada e grave, no momento em que a transfusão sanguínea torna-se indicativa no tratamento fetal, ou seja, nos casos com níveis de hemoglobina inferior a $10 \mathrm{~g} /$ $\mathrm{dL}$.

Ressalta-se que o DBVE é de fácil obtenção, uma vez que a visão de quatro câmaras do coração fetal já faz parte do exame ecográfico obstétrico básico e pode ser realizada pelo ultrasonografista em serviços de atenção primária. Estudos anteriores ainda pouco conclusivos tentaram predizer a anemia fetal por mensuração cardíaca $^{18}$. Com o uso de análise multivariada, demonstrou-se que, independente do fator fisiológico do crescimento fetal levando ao aumento concomitante das câmaras cardíacas, a redução dos niveis da hemoglobina se correlacionam com o aumento da medida do DBVE ${ }^{19}$. Esta medida representa a maior dimensão dos ventrículos no plano das valvas atrioventriculares e é um método de grande valor para a detecção da cardiomegalia fetal $^{10}$.

A remodelação ventricular com dilatação das câmaras do coração é a principal responsável pelo aumento do débito cardíaco observado nos fetos anêmicos, e provavelmente responsável pelos bons resultados do índice cárdio-femoral proposto. Outros fatores que contribuem para a manutenção do desempenho da função cardíaca de fetos com 
anemia incluem a queda do hematócrito com diminuição da viscosidade do sangue ${ }^{20}$ e o aumento do retorno venoso ${ }^{21}$, além da discreta elevação da freqüência cardíaca. Contudo, eles não parecem influenciar diretamente a medida do diâmetro biventricular externo.

$O$ índice cárdio-femoral utiliza a razão entre o DBVE e o comprimento do fêmur para compor um parâmetro independente da idade gestacional. Este índice é possivel, pois ambas são variáveis de crescimento constante e proporcional no transcurso da gravidez. Desta forma, a elevação do índice cárdio-femoral reflete a dilatação cardíaca no processo de agravamento da anemia fetal, o que é demonstrado pela correlação inversa entre o índice e a concentração da hemoglobina fetal encontrada no estudo. Contudo, é necessário afastar as causas de encurtamento de ossos longos que poderiam comprometer falsamente o índice como as displasias esqueléticas e cromossomopatias. Limitações do exame ecocardiográfico incluem o posicionamento fetal, obesidade materna e movimentação fetal excessiva.

$\mathrm{O}$ achado mais relevante deste estudo foi sua aplicabilidade clínica, pois mostra que é possivel, com o uso de medidas ecográficas, a identificação dos fetos com indicação de procedimento invasivo propedêutico e terapêutico da isoimunização materna pelo fator Rh. Quando o valor do índice cárdiofemoral é igual ou superior a 0,6 (exame alterado), observamos bons resultados de sensibilidade $(80,9 \%)$, especificidade $(83,1 \%)$, valor preditivo positivo $(73,1 \%)$ e valor preditivo negativo $(88,5 \%)$ para a detecção de fetos com hemoglobina menor que $10 \mathrm{~g} / \mathrm{dL}$, nivel ideal para realização do tratamento intra-uterino ${ }^{11}$. Portanto, acredita-se que este índice terá grande utilidade para diagnóstico da anemia fetal isoladamente ou em associação com outros métodos não invasivos, com a grande vantagem de ser metodologia simples e de custo acessivel.

\section{Referências}

1. Bowman JM. Hemolytic disease. In: Creasy RK, Resnik R, editors. Maternal-fetal medicine. 4th ed. Philadelphia: Saunders; 1999. p. 711-43.

2. Cabral ACV. Obstetrícia. 2a ed. Rio de Janeiro: Revinter; 2002. p. 203-6.

3. Divakaran TG, Waugh J, Clark TJ, Khan KS, Whittle MJ, Kilby MD. Noninvasive techniques to detect fetal anemia due to red blood cell alloimmunization: a systematic review. Obstet Gynecol. 2001;98(3):509-17.

4. Van Kamp IL, Klumper FJ, Oepkes D, Meerman RH, Scherjon SA, Vandenbussche FP, Kanhai HH. Complications of intrauterine intravascular transfusion for fetal anemia due to maternal redcell alloimmunization. Am J Obstet Gynecol. 2005;192(1):171-7.

5. Mari G, Detti L, Oz U, Zimmerman R, Duerig P, Stefos T. Accurate prediction of fetal hemoglobin by Doppler ultrasonography. Obstet Gynecol. 2002;99(4):589-93.

6. McLean LK, Hedriana HL, Lanouette JM, Haesslein HC. A retrospective review of isoimmunized pregnancies managed by middle cerebral artery peak systolic velocity. Am J Obstet Gynecol. 2004;190(6):1732-6.

7. Rightmire DA, Nicolaides $\mathrm{KH}$, Rodeck CH, Campbell $\mathrm{S}$. Fetal blood velocities in $\mathrm{Rh}$ isoimmunization: relationship to gestational age and to fetal hematocrit. Obstet Gynecol. 1986;68(2):233-6.

8. Saltzman DH, Frigoletto FD, Harlow BL, Barss VA, Benacerraf BR. Sonographic evaluation of hydrops fetalis. Obstet Gynecol. 1989;74(1):106-11.

9. DeVore GR, Mayden K, Tortora M, Berkowitz RL, Hobbins JC. Dilation of the fetal umbilical vein in rhesus hemolytic anemia: a predictor of severe disease. Am J Obstet Gynecol. 1981;141(4):464-6.

10. Rizzo G, Arduini D, Romanini C. Doppler echocardiographic assessment of fetal cardiac function. Ultrasound Obstet Gynecol. 1992;2(6):43445.

11. Barcelos, TB. Acuidade do índice ecográfico diâmetro biventricular externo/comprimento do fêmur na predição da anemia fetal em gestantes isoimunizadas [tese]. Belo Horizonte: Universidade Federal de Minas Gerais; 2003.

12. Robertson JG. Evaluation of the reported methods of interpreting spectrophotometric tracing of amniotic fluid in rhesus isoimmunization. Am J Obstet Gynecol. 1966;95(1):120-6.

13. De Vore GR, Siassi B, Platt LD. Use of femur length as a means of assessing M-mode ventricular dimensions during second and third trimesters of pregnancy in normal fetus. J Clin Ultrasound. 1985;13(9):619-25.

14. Hadlock FP. Determinação ultra-sonográfica da idade gestacional. In: Callen PW, editor. Ultrasonografia em obstetrícia e ginecologia. 3a ed. Rio de Janeiro: Guanabara Koogan; 1996. p. 82-97.

15. Nicolaides KH, Sadovsky G, Cetin E. Fetal heart rate patterns in red blood cell isoimmunized pregnancies. Am J Obstet Gynecol. 1989;161(2):3516. 
16. Dukler D, Oepkes D, Seaward G, Windrim R, Ryan G. Noninvasive tests to predict fetal anemia: a study comparing Doppler and ultrasound parameters. Am J Obstet Gynecol. 2003;188(5):1310-4.

17. Nishie EN, Brizot ML, Liao AW, Carvalho MH, Toma $\mathrm{O}$, Zugaib M. A comparison between middle cerebral artery peak systolic velocity and amniotic fluid optical density at $450 \mathrm{~nm}$ in the prediction of fetal anemia. Am J Obstet Gynecol. 2003;188(1):214-9.

18. Ouzounian JG, Monteiro HA, Alsulyman OM, Songster GS. Ultrasonographic fetal cardiac measurement in isoimmunized pregnancies. J Reprod Med. 1997;42(6):342-6.
19. Rodrigues RLM, Pereira AK, Taveira MR, Melo IG, Osana GC, Cabral ACV. Avaliar a correlação entre a concentração da hemoglobina e a medida ecográfica do diâmetro biventricular externo em fetos anêmicos de gestantes isoimunizadas. Arq Bras Cardiol. 2005;84(5):393-6.

20. Fumia FD, Edelstone DI, Holzman IR. Blood flow and oxygen delivery to fetal organs as functions of fetal hematocrit. Am J Obstet Gynecol. 1984;150(3):274-82.

21.Kirkinen P, Jouppila P, Eik-Nes S. Umbilical vein blood flow in rhesus-isoimmunization. $\mathrm{Br} \mathrm{J}$ Obstet Gynaecol. 1983;90(7):640-3. 